\title{
MULTI-COLOR IMAGING OF SELECTED SOUTHERN INTERACTING GALAXIES
}

\author{
Eric P. Smith and Paul Hintzen \\ Laboratory for Astronomy and Solar Physics \\ Goddard Space Flight Center \\ Greenbelt, MD 20771 USA
}

\section{Introduction}

We present preliminary results from a study of selected Arp-Madore Southern Hemisphere peculiar galaxies. Broadband CCD images ( $B V R I)$ of a subset of these galaxies allow us to study each galaxy's optical morphology, color, and (in a crude manner) degree of nuclear activity, and to compare them with similar data we possess on other active galaxies. Many of these galaxies have optical morphologies closely resembling those of powerful radio galaxies (Smith and Heckman 1989), yet their radio emission is unremarkable. Accurate positions for subsequent spectroscopic studies have been determined along with broad band photometry and morphology studies. Detailed observations of these comparatively bright, low-redshift, well-resolved interacting systems should aid our understanding of the role interactions play in triggering galaxy activity. This work is the initial effort in a long term project to study the role played by the dynamics of the interaction in the production and manifestations of activity in galaxies, and the frequency of galaxy mergers.

\section{Preliminary Results}

Galaxies were selected from the Catalogue of Southern Peculiar Galaxies and Associations (Arp and Madore 1987) based upon their morphology. We specifically chose galaxies from category 15 "Loops, Tails, and Debris" (many of which are also listed in category 2, "Interacting Doubles") - because we were looking for those systems which contained evidence for, or suggestions of interacting spiral galaxies. A total of 31 systems were imaged on 14-17 March 1989 with the CTIO 4-meter using a TI CCD and the standard $B, V, R, I$ filter set. The data were bias-subtracted and flat fielded using standard procedures, and $R$ and $I$ frames subsequently corrected for fringing using images of blank sky fields. Before performing our photometry we carefully edited each frame to remove stellar images which overlay or were close to the galaxy images. The details of this method may be found in Smith and Heckman (1989). We performed synthetic aperture photometry, and found the agreement between our measurements and previous 
photometry to be very good (better than $0.1 \mathrm{mag}$ ). Magnitudes have been corrected for Galactic reddening using the HI maps of Burstein and Heiles (1982).

We are in the midst of our data analysis and therefore the results we discuss are preliminary. In figure 1 we present two representative galaxjes from our sample. The obvious damage these galaxies have sustained penetrates deep within their isophotal structure, not being restricted to low surface brightness distortions. This suggests that these are recent mergers or interactions which have not had time to virialize even their densest regions. For the system $0642-801$ we find that the small galaxy to the west have colors and a surface brightness profile like those of a normal elliptical. The morphologically peculiar galaxy has colors which are blue by about $0.1 \mathrm{mag}$ (in $(B-V)$ relative to a normal elliptical. This system was detected by IRAS in the point source survey at $60 \mu \mathrm{m}$ $\left(f_{60 \mu \mathrm{m}}=0.68 \mathrm{Jy}\right)$. The second system, $1255-430$ is extremely peculiar, and may possess multiple nuclei though spectroscopy is needed for confirmation. This galaxy was not detected by IRAS in the point source survey and has colors typical of an Sb galaxy $((B-V)$ $=0.77$ ).

Morphologically, the sample is (not surprisingly!) dominated by galaxies which display one or more tails (extended features whose length/width $\geq 3$ ) with 19 out of 31 systems having these types of features. More importantly, 17 of the systems clearly display multiple intensity peaks within the main luminous body (some of which may be caused by dust lanes) or are clearly two galaxies embedded in a common envelope. Spectra of these objects will be studied to investigate the detailed kinematics of the interactions. The galaxies in our sample display a wide range of color index values (figure 2) and color index gradients. These colors and gradients are strongly influenced by dust present in these galaxies (35\% of the sample objects are listed in the IRAS Point Source Catalog), and the likely presence of nonstellar emission-lines and bursts of star formation. Until spectra (or narrow band images) are obtained we cannot separate nonstellar emission from the underlying stellar continuum emission. We have, along with $N$. Kassim (NRL), observed six of the objects from our full sample ( $\sim 80$ systems) with the VLA. Three objects were detected ( $\sim 10 \mathrm{mJy}$ level) while the three others were not. Two of the systems detected do not show any radio structure while the third does appear to be resolved.

\section{References}

Arp, H. C., and Madore, B. F. 1987, A Catalogue of Southern Peculiar Galaxies and Associations (Cambridge University Press).

Burstein, D. A., and Heiles, C. 1982, A. J., 87, 1165.

Smith, E. P., and Heckman, T. M. 1989, Ap.J.Suppl., 68, 365. 


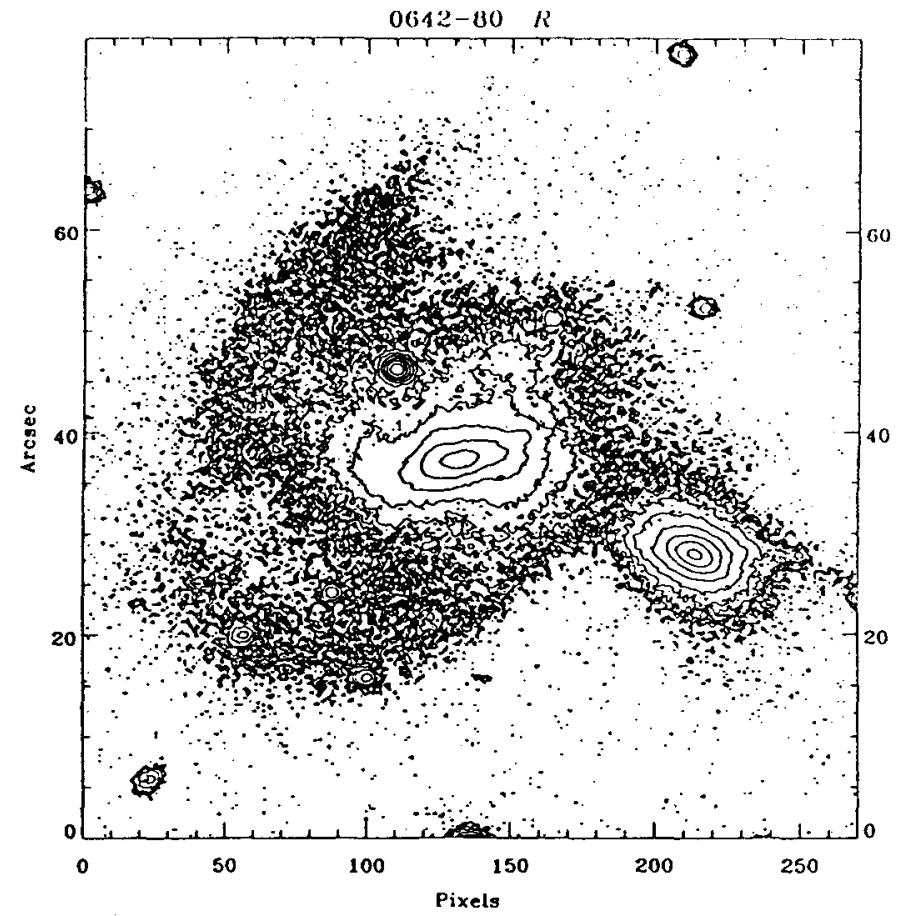

Contours [ 22.86, 22.11, 21.35, 20.60, 19.85, 19.09, 18.34, 17.59, 16.84, 16.08, 15.33]

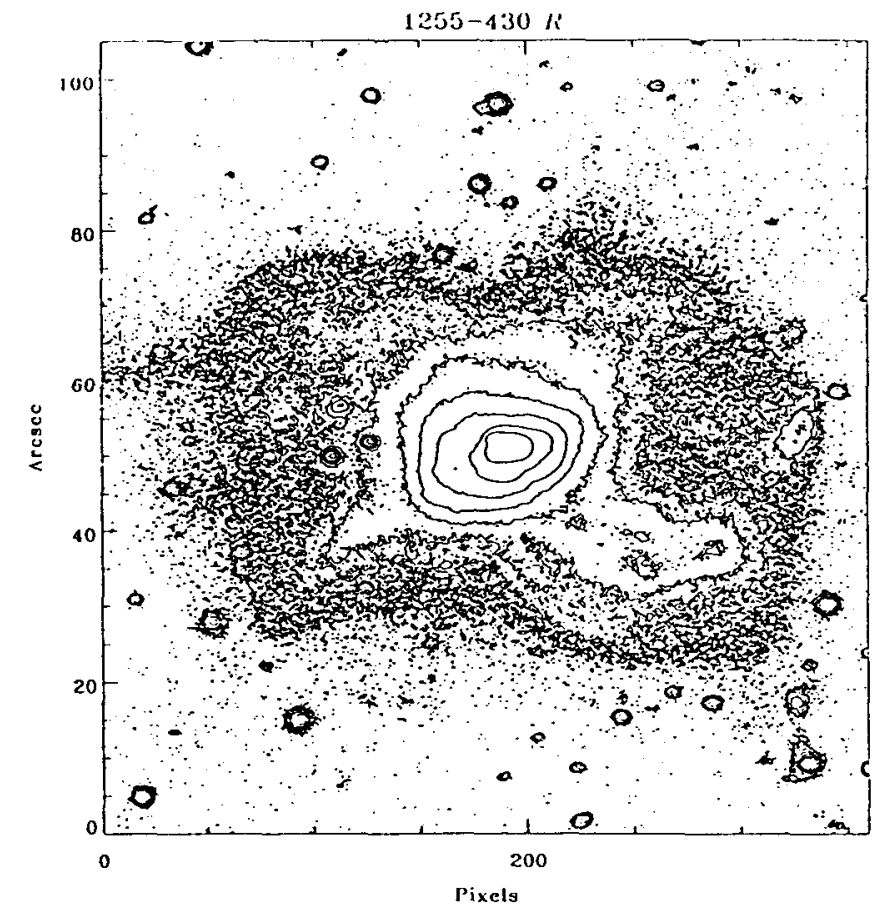

Contours | 23.64, 22.89. 22.14. 21.39. 20.63. 19.88. 19.13. 18.37. 17.62. 16.87, 16.12

Figure 1: Contour images (in $R$ with North on top, East to the left) for two of the galaxies in the sample $0642-801$ and $1255-430$. Contour levels in $R$ magnitudes/arcsec ${ }^{2}$ are listed below each plot. Images were edited to remove stars prior to synthetic aperture photometry. 

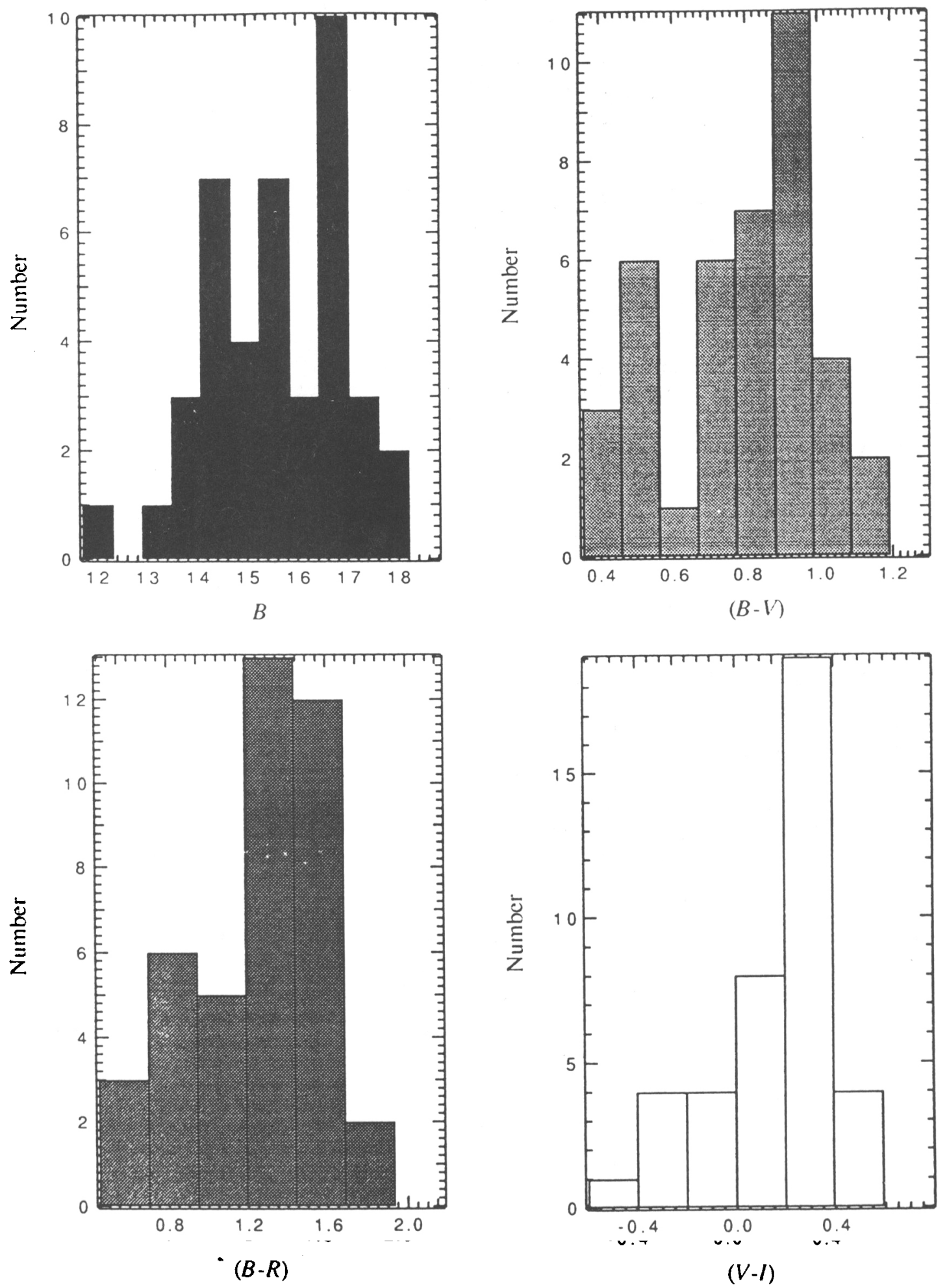

Figure 2: Distribution of $B$ magnitudes and color indices for the sample galaxies. Apparent magnitudes and colors for closely overlapping galaxies taken from apertures with radii smaller than the galaxy separation. 